\title{
El estado de la formación superior y la investigación académica en Psicología en Costa Rica. Periodo 2013-2014.
}

\section{The Art of University Education and Academic Research in Psychology in Costa Rica. 2013-2014 Period}

Fecha de recepción: 04/11/2014

Fecha de aceptación: 26/06/2015

\author{
${ }^{1}$ Alfonso Villalobos-Pérez, ${ }^{2}$ Cristina Jungue, \\ ${ }^{3}$ Alejandro Monge-Salazar y ${ }^{4}$ Kattia Vargas-Fallas. \\ 'Caja Costarricense de Seguro Social, San José, Costa Rica. \\ ${ }^{2}$ Colegio Profesional de Psicólogos de Costa Rica. \\ ${ }^{3}$ Colegio Profesional de Psicólogos de Costa Rica. \\ ${ }^{4}$ Universidad Nacional.
}

\section{resumen/abstract:}

La Psicología costarricense comenzó su desarrollo histórico en la década de los años 1930. Posteriormente, ya entrada la segunda mitad del siglo XX, se establecieron procesos académicos que desembocaron en la aparición de la primera Escuela de Psicología: En los años ochenta y noventa se expandieron dichas academias en diversas universidades privadas y públicas. Este estudio se orientó a revisar el estado de la formación en la disciplina y las condiciones de investigación en esta ciencia en Costa Rica. Para ello, se aplicaron cuestionarios a escuelas, centros de investigación y al ente regulador de la psicología nacional. Se recibieron pocas respuestas (dos cuestionarios de 21 universidades que imparten la carrera y dos centros de investigación en psicología y ciencias afines); sin embargo, se pudo constatar que la Psicología costarricense ha construido un sentido académico orientado a la formación y con menor peso en la investigación académica. La universidad que tiene mayor tradición cuenta con mejores sistemas de financiamiento y personal capacitado en el extranjero. Otras instituciones de educación están comenzando una aproximación particular a la investigación. Es de esperar que los procesos de acreditación nacionales permitan y faciliten un panorama más positivo en los próximos años para la formación y la academia psicológica costarricense en general.

Psychology in Costa Rica started its historic development in the 1930's. After this date, in the second part of the $20^{\text {th }}$ Century, academic processes were established which influenced the upcoming of the first Psychology Department: In the 80's and 90's, these academic units expanded into a diverse amount of private and public universities. This study was oriented in revising the State of Art of formation in this discipline and the conditions of research of this science in Costa Rica. For this purpose, questionnaires were applied to departments, research centers, and regulatory organs of national Psychology. For receiving little feedback on questionnaires (two questionnaires answered of 21 universities which impart Psychology and two psychological and related sciences research centers); anyhow, it resulted possible to ascertain that Costa Rican Psychology has constructed an academically oriented training with minor weight on academic research. The university that counts with major tradition also draws benefits from a better financing system and qualified personal formed abroad. Other educational institutions are starting an approximation to research. It is to be awaited that national accreditation processes allow and facilitate a rather positive panorama for the Costa Rican academic development and education in Psychology in the upcoming years in general.

\section{palabras clave/keywords:}

Costa Rica, Psicología, investigación académica, formación superior, universidad. Costa Rica, Psychology, Academic Research, Superior Education, University. 


\section{El surgimiento de la Psicología en Costa Rica}

En Costa Rica, el surgimiento de la Psicología estuvo ligado en sus inicios de forma indirecta al interés particular de los educadores del país en temas como el desarrollo y la personalidad del niño. Este interés lleva a que en el año 1924 la profesora Lilia Ramos organice la primera "escuela para padres" que tuvo como propósito el estudio del desarrollo y la personalidad del niño (Adis, 1994).

Las primeras experiencias con profesionales en psicología se dieron en la década de 1930, cuando profesionales provenientes de Chile y Puerto Rico vinieron a Costa Rica a trabajar en la elaboración y estandarización de pruebas para la medición psicológica, utilizadas posteriormente por docentes escolares. La falta de interés y el rechazo por parte de los educadores hacia la utilización de tests provocó que el trabajo realizado por estos profesionales extranjeros se perdiera (Thomas, 1979).

Es hasta el año 1940 cuando profesionales costarricenses provenientes de Chile, formados en educación, empezaron a incluir dentro de su léxico conceptos psicológicos aplicados a la educación (Thomas, 1979). Estos primeros contactos con la psicología, propiciaron la apertura de espacios dentro del currículo de la formación universitaria en la enseñanza media.

En la decada de los años 1950, se impartieron en la Escuela de Pedagogía de la Universidad de Costa Rica cursos de Psicología del niño y del adolescente, así como cursos de Higiene y salud mental dirigidos a docentes (Adis, 1994).

Inicialmente, profesores de Castellano, Estudios sociales y Religión fueron los encargados de dar las lecciones de Psicología a estudiantes de IV y V año de secundaria. Al no existir documentación bibliográfica, profesores como Isaac Felipe Azofeifa, quien se encargaba de impartir lecciones en el Liceo de Costa Rica y Teodoro Olarte en el Colegio Seminario, desarrollaron manuales de psicología que se utilizaron para la formación de los estudiantes (Thomas, 1979).

Asímismo, en 1950, gracias al entonces llamado Comité Nacional de Salud Mental, profesionales de otras ciencias empezaron a realizar capacitaciones dirigidas a maestros y al público en general que tenían como propósito informar sobre los principios y aportes de la psicología, como p.ej., la salud fisica y mental del niño, el adolescente y los adultos.

Debido a la ausencia de un espacio académico en el que pudiera iniciarse la formación profesional en psicología y a la poca presencia de psicólogos en Costa Rica en estos años, los temas inherentes al quehacer psicológico fueron desempeñados por profesionales de otras áreas del conocimiento, entre ellos pedagogos, psiquiatras y trabajadores sociales (Thomas, 1979).

El Dr. Gonzalo Adís Castro fundó en el año 1957 el Servicio de Psicología Clinica del Hospital Nacional Psiquiatrico. Este hecho representó el primer trabajo en el campo clínico a nivel hospitalario de profesionales en Psicología. Dentro de las primeras tareas que se desarrollaron estaba la traducción de algunas pruebas psicológicas, como p.ej., el Multifásico de Personalidad de Minnesota, el Mini-Mult, los Tests de Wechsler-Bellevue y el Test de Oraciones Incompletas, entre otros (Adis, 1991). Asimismo, se desarrollaron programas de intervención y tratamiento individual y para parejas.

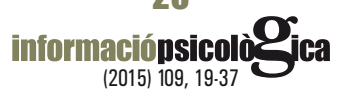




\section{La Psicología como ciencia en Costa Rica}

En la década de 1960, el regreso de psicólogos formados en los Estados Unidos y Europa provocó que se iniciara el trabajo por el reconocimiento de la Psicología como ciencia. Estos esfuerzos propiciaron que en 1961 se creara el Centro de Investigaciones Psicológicas en la Sección de Ciencias del Hombre de la Facultad de Ciencias y Letras de la Universidad de Costa Rica (primera universidad de enseñanza pública de Costa Rica, después del cierre de la Universidad de Santo Tomás en 1888) como primer espacio académico científico de la Psicología en el país (Thomas, 1979).

El centro, inicialmente, se encargó de realizar las gestiones iniciadas por el entonces llamado Comité de Evaluación. Posteriormente, además de estas tareas se incluyó el desarrollo de investigaciones de gran importancia, como p.ej., la creación de una escala nacional centrada en las actitudes de las personas hacia quienes en esa época se les denominaba "enfermos mentales" (Adis, 1994).

Cinco años después de la creación del Centro de Investigaciones Psicológicas, se inició la formación de psicólogos a nivel de bachillerato universitario. Por primera vez en Costa Rica se abrió un espacio para la formación profesional en Psicología bajo la responsabilidad de psicólogos (Thomas, 1979). En 1969, el Centro de Investigaciones Psicológicas pasó a llamarse Instituto de Investigaciones Psicológicas y adquirió con esta transformación una estructura científica y jurídica que permitió que el instituto tenga hasta hoy un papel importante en el campo académico-científico de Costa Rica (Adis, 1994).

\section{El sistema de universidades privadas vs. públicas}

Durante el gobierno del Presidente de la República José Figueres Ferrer, se creó en la ciudad de Heredia la Universidad Nacional. Esta nueva casa de enseñanza universitaria pública acogió a los profesionales de las extintas Escuela normal y Escuela normal superior. En esta nueva institución de educación superior universitaria se creó el Departamento de Psicología, en el que, debido a las instrucciones giradas por la Oficina de Planificación de la Educación Superior (OPES) del Consejo Nacional del Rectores (CONARE), no se pudieron formar profesionales, al considerar que era poca la demanda que había en el país. No fue hasta finales de 1990 que inició la formación profesional de psicólogos en esta universidad (Cordero, Dormond \& Flores, 2000).

En 1975, bajo los lineamientos del III Congreso Universitario de la Universidad de Costa Rica, se creó el Sistema de Estudios de Posgrado (UCR-SEP) con el objeto de propiciar y diseminar el desarrollo cientifico e investigativo en la Universidad de Costa Rica (Diaz \& Diaz, 2014). A partir del año 1984, inició la Maestría en Psicología con énfasis en Metodología de la Investigación Psicológica, el primer posgrado en Psicología en Costa Rica. Y en 1976, se creó en la Universidad de Costa Rica la primera Escuela de Psicología de Costa Rica, que fue por algunos años la única casa de estudio en la que se brindó este tipo de formación profesional.

En 1977, se creó el Colegio Profesional de Psicólogos de Costa Rica, institución de orden público y administración privada, que hasta la fecha se ha encargado de agrupar y regular el ejercicio profesional de los profesionales en psicología.

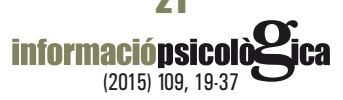


La restricción impuesta por la Oficina de Planificación de la Educación Superior, en cuanto al cupo de estudiantes en algunas de las carreras de mayor demanda en Costa Rica, propició la creación de la primera universidad privada de Costa Rica: la Universidad Autónoma de Centroamérica (UACA). Así, en 1981 existieron dos universidades privadas que impartieron la carrera de Psicología en Costa Rica: la UACA y la Universidad Autónoma Monterrey.

Debido a las presiones de grupos con poder dentro de las principales centros de educación universitaria pública, se creó, a partir del año 1988, el Consejo Nacional de Enseñanza Superior Universitaria Privada (CONESUP), ente que hasta la fecha se encarga de regular la modificación y la creación de las universidades privadas.

En la década de 1990, se contabilizaron en el país nueve universidades privadas aprobadas por el CONESUP para impartir la carrera de Psicología en los distintos niveles de la educación superior universitaria: bachillerato, licenciatura y maestría (Cordero, Dormond y Flores, 2000). Este número casi dobló para el año 2012 a 19 universidades privadas aprobadas para impartir la carrera de Psicología en los niveles de bachillerato, Licenciatura y Maestría en Costa Rica (CONESUP, 2012).

El Cuarto Informe del Estado de la Educación (2013) contabilizó para el año 2011 un total de 37 carreras aprobadas de Psicología en los niveles de bachillerato, licenciatura y maestría. Del total, nueve carreras se encontraron en universidades estatales y las 28 restantes se encontraron distribuidas en las universidades privadas aprobadas por el CONESUP.

Debido al gran número de universidades privadas que se crearon en Costa Rica y a la necesidad de asegurar la calidad de los procesos educativos que las instituciones de educación superior universitaria impartieron, el Gobierno de la República fundó en el año 2002 el Sistema Nacional de la Educación Superior (SINAES), en el cual se encontraron acreditadas en el año 2014 únicamente tres universidades: la Universidad de Iberoamerica, la Universidad Católica de Costa Rica y la Universidad de Costa Rica.

Lo anterior invita a reflexionar en torno a la calidad y cantidad de profesionales que las universidades públicas y las privadas actualmente gradúan en Costa Rica. Según datos de la División de Sistemas de la OPES, el CONARE y el CONESUP (2013), durante el período comprendido entre los años 2000-2013 hubo un crecimiento sostenido en la cantidad de profesionales que se graduaron en alguna de las carreras ubicadas dentro de las Ciencias sociales, a la cual pertenece la Psicología en Costa Rica. Hasta el año 2012 se graduaron en Costa Rica 160.150 profesionales en el área de las Ciencias sociales. Solamente en el año 2013 se graduaron 19.796 profesionales en las carreras de las Ciencias sociales, de ese total 14.569 correspondieron a los estudiantes graduados en universidades privadas y los restantes 5.227 en universidades estatales.

Según consta en el documento "Seguimiento de la condición laboral de las personas graduadas 2000-2007 de las universidades costarricenses" (Consejo Nacional de Rectores de Costa Rica, 2010), el 93,04\% de las personas graduadas en Costa Rica tuvieron un empleo, mientras un 6,97\% se encontraron desempleados. En el análisis por áreas del conocimiento, se muestra que los profesionales graduados en Ciencias Sociales ocupan el cuarto lugar en cuanto a los porcentajes de mayor desempleo de las personas graduadas, por área de estu-

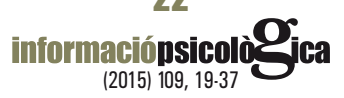


dio, a saber Ciencias Básicas (3,11\%), Artes y Letras (2,58 \%), Recursos Naturales (2,48\%) y Ciencias Sociales $(2,42 \%)$. Las áreas de Ciencias de la Salud e Ingeniería ocupan los primeros lugares en empleabilidad por área de estudio.

Los registros de Colegio Profesional de Psicólogos de Costa Rica (CPPCR), proporcionan información complementaria a lo descrito en el párrafo previo, de esta forma la Figura 1 que, a partir de 2000, se observa un patrón de incremento en las incorporaciones, que tienen como punto de inicio el 2004 y que, al 2013, aún se mantiene por encima de las 500 incorporaciones anuales.

Partiendo de ese contexto, se planteó como propósito de este estudio la descripción del estado de la educación superior y la investigación académica en Costa Rica, durante el periodo 2013-2014..

Figura 1. Cantidad de profesionales que se incorporaron al CPPCR por año

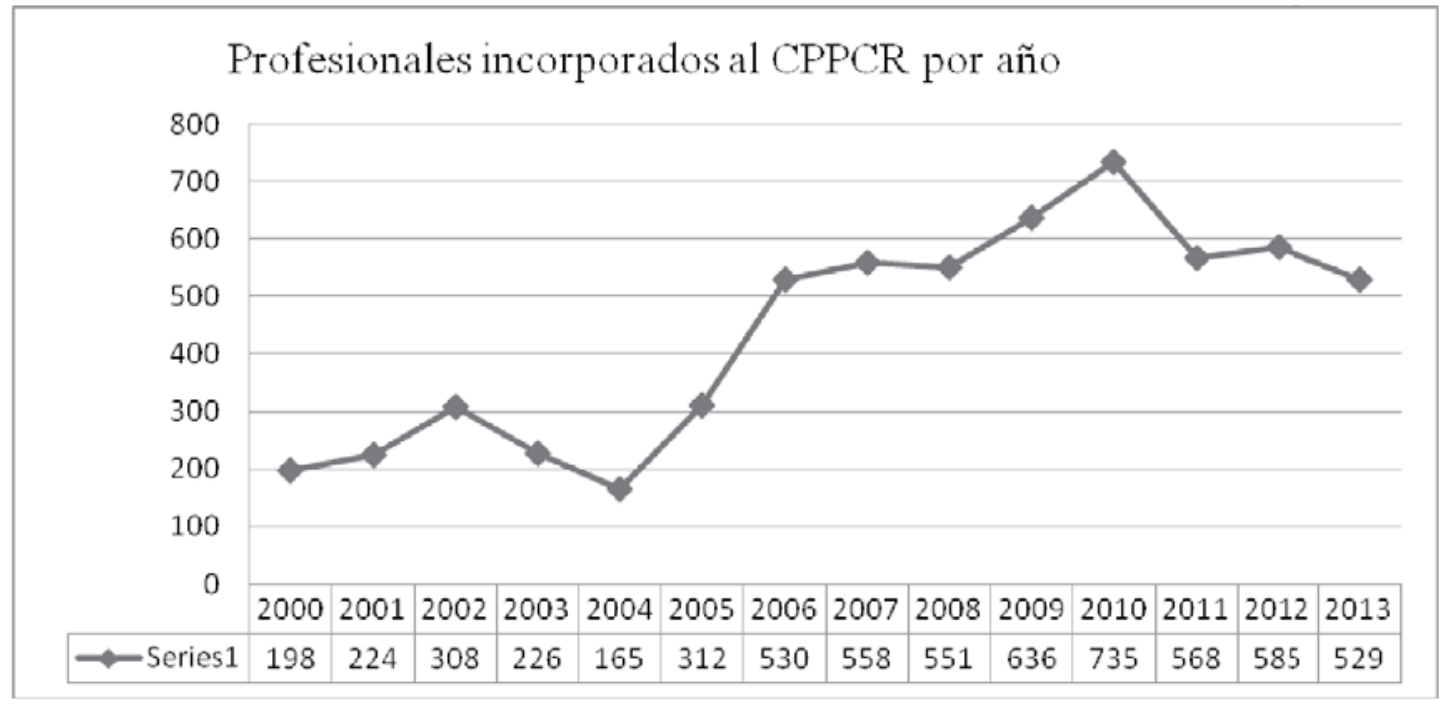

FuFuente: Datos del Colegio Profesional de Psicólogos de Costa Rica (CPPCR).

\section{Método}

\section{Tipo de estudio}

El desarrollo de la investigación tuvo un alcance de carácter descriptivo, con el fin de establecer el panorama de la situación actual de la formación académica en Psicología y de la investigación científica en la disciplina, según datos oficiales de varias fuentes acreditadas a nivel nacional, bajo tres ejes: (a) docencia: actividades de formación e investigación, (b) academia: acciones investigativas y organización de proyectos de investigación y (c) gestión-rectoría: gestión y rectoría de procesos de investigación por el ente rector (Colegio Profesional de Psicólogos de Costa Rica [CPPCR]). 


\section{Sujetos}

La unidad de análisis fueron las instituciones académicas que impartieron Psicología y que realizaban investigación científica en el momento de la investigación. En la tabla 1 se presenta la descripción del conjunto de datos analizados.

Tabla 1. Caracterización de los tipos de universidades que imparten psicología en Costa Rica y que presentan centros e institutos de investigación académica.

\begin{tabular}{|c|c|c|}
\hline & \multicolumn{2}{|c|}{ Tipo de universidad } \\
\hline & Pública & Privada $^{\text {a }}$ \\
\hline Cantidad total de universidades & 5 & 53 \\
\hline $\begin{array}{l}\text { Cantidad de universidades que imparten } \\
\text { psicología (grado y posgrado) }\end{array}$ & 2 & 19 \\
\hline $\begin{array}{l}\text { Cantidad de universidades a las que se les } \\
\text { solicitó información }\end{array}$ & 2 & 4 \\
\hline $\begin{array}{l}\text { Cantidades de universidades que participaron } \\
\text { (enviaron respuestas) en el estudio }{ }^{b, c}\end{array}$ & 1 & 1 \\
\hline $\begin{array}{l}\text { Cantidad de centros o institutos relacionados } \\
\text { con psicología (de adscripción universitaria), } \\
\text { que participaron en el estudio }\end{array}$ & 2 & - \\
\hline $\begin{array}{l}\text { Nota. }{ }^{\text {a }} \text { Datos extraídos del Consejo Superior d } \\
\text { go.cr/sites/default/files/page/adjuntos/universi } \\
\text { sidad privada envío información, pero esta pre } \\
\text { efectos de este estudio no fue contabilizada en } \\
\text { administra su grado y posgrado de manera dife }\end{array}$ & $\begin{array}{l}\text { ón (CONE } \\
\text { adas-auto } \\
\text { lo artícul } \\
\text { La univers }\end{array}$ & $\begin{array}{l}\text { http://www.mep. } \\
\text { segunda univer- } \\
\text { dexadas, para } \\
\text { envió información, }\end{array}$ \\
\hline
\end{tabular}

La tabla 1 permite observar el comportamiento general de las universidades (tanto públicas como privadas) que participaron en el estudio. Del total de sistemas universitarios aprobados a nivel país (58), 21 de ellas imparten psicología. El estudio contó con la colaboración de dos grados (universidad pública y privada) y un posgrado (universidad pública). En relación con entes oficiales y profesionales (que tienen personal dedicado a este ámbito de acción en psicología) de investigación, enviaron respuestas dos: el Centro de Investigación en Neurociencias (CIN) y el Instituto de Investigaciones Psicológicas (IIP), ambos de la Universidad de Costa Rica (UCR). La única universidad privada que proporcionó datos fue la Universidad de Iberoamérica (UNIBE).

Se solicitó información al Colegio Profesional de Psicólogos de Costa Rica (CPPCR), sobre el estado de la psicología en Costa Rica, así como una serie de variables relacionadas al tema de este estudio (por ejemplo cantidad y tipo de posgrados en las diversas universida-

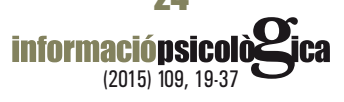


des públicas y privadas), sin embargo, al momento de la solicitud de estos datos, se indicó a los autores de este manuscrito que el CPPCR no mantenía información específica sobre el estado de los posgrados y sus enfoques de formación.

\section{Criterios de inclusión}

Criterios de inclusión fueron la anuencia a participar por parte de programas acreditados o grupos de investigación activos con programas, institutos o centros y con personal de Psicología activos, con la producción de artículos incluidos en revistas científicas indexadas y con cursos de investigación en sus currículos académicos.

\section{Categorías de análisis}

Los datos se sintetizaron en tres grupos de información:

Docencia: referido a las actividades de formación e investigación que se realizan dentro de las escuelas o facultades de psicología de las universidades consultadas.

Académico: se incluyeron aquí las acciones investigativas y de organización de proyectos de investigación, que se realizan en institutos o centro de investigación de las universidades consultadas.

Gestión-rectoría: describió las actividades vinculadas a la gestión de los procesos de investigación documentadas en el CPPCR.

\section{Instrumentos}

Con el fin de capturar información para abordar el estado de la formación y la investigación en Costa Rica, se crearon tres encuestas, que incluían preguntas abiertas y cerradas referentes a: la formación en las Escuelas de Psicología, los programas de investigación científicos en Psicología y la información consignada por el Colegio Profesional de Psicólogos de Costa Rica.

Los ejes de las preguntas fueron: años de existencia de la entidad, cantidad de artículos científicos publicados, cantidad de docentes/ investigadores, financiamiento de los proyectos, regulación de la investigación y vínculos entre los docentes, estudiantes y temas de investigación.

\section{Procedimiento}

En primer lugar se solicitó permiso a las direcciones de las diferentes Escuelas de Psicología, (direcciones de) programas de investigación y al ente rector de la Psicología costarricense (CPPCR), para el llenado de varios instrumentos de recolección de datos.

En un segundo momento se entregaron los tres instrumentos de recolección de datos a los diferentes entes académicos que aceptaron participar.

Posteriormente (tercer lugar), se solicitó información al Registro Científico y Tecnológico de Costa Rica (RCT) para determinar la actualización de las investigaciones psicológicas en Costa Rica (los datos no se incluyeron, debido a que el más reciente correspondió al año 2001, según el RCT esto ocurrió como consecuencia del atraso y fallos en la actualización de los registros científicos de este organismo nacional).

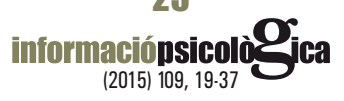


En un cuarto lugar, los instrumentos se analizaron en tablas correspondientes a cada categoría de investigación.

Por último (quinto lugar), se revisaron los currículos académicos de ocho universidades, con el fin de observar la cantidad y ubicación de los cursos formativos en investigación.

\section{Resultados}

En las siguientes páginas se exponen los principales resultados obtenidos del conjunto de instrumentos, que fueron respondidos por los diferentes participantes de este estudio. Es importante destacar que, como se puede observar de los datos sistematizados en la tabla 1, a pesar de que el país cuenta con 21 universidades que imparten psicología, solo se pudo recolectar información de ellas y únicamente dos entes de investigación profesional psicología participaron.

Un gran número de estas universidades que forman en psicología, no realizan investigación académica profesional (no tienen grupos, programas, líneas, institutos o centros organizados y con personal de planta y, por tanto, no pueden publicar resultados de investigación en revistas científicas indexadas internacionales) y, la información expuesta se refiere solo a las que sí están desarrollando toda una logística y tradición investigativa formal a nivel país. .

\section{Datos docencia}

La Universidad de Costa Rica (pública) respondió desde dos instancias: la Escuela de Psicología (UCR-EP) y el Posgrado (UCR-SEP) con 48 y 31 años de existencia, respectivamente, y la Universidad de Iberoamérica (UNIBE, privada) respondió desde su Escuela de Psicología (con 16 años de existencia). Esta información se extrajo de las respuestas al ítem 1 del cuestionario respectivo.

Es importante señalar que UCR-EP posee la mayor cantidad de docentes que producen investigación y, además son patrocinados por su unidad académica (algunos/as de ellos/ as también son investigadores/as del IIP y del CIN), esto no es una práctica común en la UNIBE.

Las tres unidades académicas (UCR-EP, UCR-SEP y UNIBE), manifestaron pasar sus proyectos de investigación por un comité de ética previo.

Como fuentes de financiamiento para la totalidad de los proyectos de investigación, la UCR nombró a la Vicerrectoría de Investigación y la Escuela de Psicología, mientras que los proyectos de UNIBE son financiados por su universidad.

Cuando se consultó si los trabajos finales de graduación (TFG) desarrollados por estudiantes (de grado y posgrado), concluían en un artículo enviado a revistas indexadas, se pudo encontrar que existía una razón de artículos indexados/promedio de estudiantes año con la siguiente distribución: UCR-SEP $=2 / 10: 76 / 100$, UNIBE $=2 / 10: 51-75$ y UCR-EP no proporcionó datos sobre este rubro de artículos indexados. Los artículos producidos por los graduados recibieron en la UNIBE y en la UCR-EP asistencia técnica del docente. 
Tabla 2.- Datos sobre tres Escuelas de Psicología en Costa Rica, 2014, que respondieron la solicitud

\begin{tabular}{|c|c|c|c|}
\hline Ítem & $\begin{array}{l}\text { Universidad de Costa Rica, } \\
\text { Posgrado (UCR-SEP) }\end{array}$ & $\begin{array}{l}\text { Universidad de } \\
\text { Iberoamérica (UNIBE), } \\
\text { Escuela de Psicología }\end{array}$ & $\begin{array}{l}\text { Universidad de Costa } \\
\text { Rica, Escuela de Psi- } \\
\text { cología (UCR-EP) }\end{array}$ \\
\hline $\begin{array}{l}\text { 2. Promedio estudiantes por año o periodo } \\
\text { lectivo. }\end{array}$ & Entre $76-100$ & Entre $51-75$ & Más de 101 \\
\hline $\begin{array}{l}\text { 3. Cantidad de docentes que dictan leccio- } \\
\text { nes en escuela/posgrado. }\end{array}$ & Entre $11-25$ & Entre $11-25$ & Entre $51-75$ \\
\hline \multirow{3}{*}{$\begin{array}{l}\text { 4. Nivel académico máximo/cantidad por } \\
\text { grado académico (docentes de la univer- } \\
\text { sidad). }\end{array}$} & \multirow{3}{*}{$\begin{array}{l}18 \text { Maestría acadé- } \\
\text { mica } \\
3 \text { Doctorado }\end{array}$} & \multirow{3}{*}{$\begin{array}{l}10 \text { Licenciatura } \\
16 \text { Maestría profe- } \\
\text { sional }\end{array}$} & 10 Licenciatura \\
\hline & & & $\begin{array}{l}25 \text { Maestría } \\
\text { académica }\end{array}$ \\
\hline & & & 26 Doctorado \\
\hline 5. Existencia de régimen académico activo. & Sí & No & Sí \\
\hline $\begin{array}{l}\text { 6. Número de docentes inscritos en el } \\
\text { régimen. }\end{array}$ & Menos de 10 & N/A & Entre $26-50$ \\
\hline $\begin{array}{l}\text { 7. Cantidad de docentes que producen } \\
\text { investigación con patrocinio de unidad } \\
\text { académica. }\end{array}$ & Menos de 10 & Menos de 10 & Entre $11-25$ \\
\hline $\begin{array}{l}\text { 8. Cantidad de proyectos de investigación } \\
\text { activos dentro de unidad académica. }\end{array}$ & Entre $1-5$ & Entre $1-5$ & Entre $11-15$ \\
\hline $\begin{array}{l}\text { 9. Cantidad de artículos de los proyectos de } \\
\text { investigación }\end{array}$ & Entre 2-10 & Entre 2-10 & No contesta \\
\hline $\begin{array}{l}\text { 10. Cantidad de artículos aceptados en } \\
\text { revistas indexadas. }\end{array}$ & Entre 2-10 & No contesta & No contesta \\
\hline $\begin{array}{l}\text { 11. Cantidad de simposios/congresos/ } \\
\text { conferencias con presentación de resultados } \\
\text { de investigación. }\end{array}$ & Entre 6-10 & Entre 2-5 & No contesta \\
\hline $\begin{array}{l}\text { 12. Investigaciones pasan por filtraje de } \\
\text { comité académico (comité ético científico). }\end{array}$ & Sí & Sí & Sí \\
\hline $\begin{array}{l}\text { 13. Fuente que provee de financiamiento en } \\
\text { investigación. }\end{array}$ & $\begin{array}{l}\text { Vicerrectoría de in- } \\
\text { vestigación (UCR) }\end{array}$ & No contesta & $\begin{array}{l}\text { Escuela de } \\
\text { Psicología }\end{array}$ \\
\hline $\begin{array}{l}\text { 14. Del total de proyectos activos, cantidad } \\
\text { que han sido financiados por lo indicado en } \\
\text { Respuesta } 13 \text {. }\end{array}$ & Entre $1-5$ & No contesta & Entre $11-15$ \\
\hline $\begin{array}{l}\text { 15. Monto aprox. del financiamiento recibi- } \\
\text { do para proyectos de investigación activos. }\end{array}$ & $\begin{array}{l}\text { 7,000,000 Colones } \\
(13,000 \text { USD) }\end{array}$ & $\begin{array}{l}\text { 500,000 Colones } \\
\text { (920 USD) }\end{array}$ & No contesta \\
\hline $\begin{array}{l}\text { 16. Cantidad de trabajos finales de gradua- } \\
\text { ción (TFG) producidos por estudiantes. }\end{array}$ & Entre $11-15$ & Mayor a 36 & Entre $31-35$ \\
\hline $\begin{array}{l}\text { 17. Cantidad de artículos derivados de los } \\
\text { TFG. }\end{array}$ & Entre 2-10 & Entre 2-10 & No contesta \\
\hline $\begin{array}{l}\text { 18. Cantidad de artículos derivados de TFG } \\
\text { incluidos en revistas indexadas. }\end{array}$ & Entre 2-10 & No contesta & No contesta \\
\hline $\begin{array}{l}\text { 19. Los TFG se incluyen en los proyectos } \\
\text { de investigación consolidados en la unidad } \\
\text { académica. }\end{array}$ & Sí & No & Sí (la minoría) \\
\hline $\begin{array}{l}\text { 20. Los TFG pasan por un comité ético } \\
\text { científico o equivalente. }\end{array}$ & Sí & No & Sí \\
\hline $\begin{array}{l}\text { 21. Los artículos producidos por los } \\
\text { graduados reciben asistencia técnica del } \\
\text { docente. }\end{array}$ & No & Sí & $\begin{array}{l}\text { Sí (no es obliga- } \\
\text { toria) }\end{array}$ \\
\hline
\end{tabular}




\section{Datos académicos}

El Centro de Investigación en Neurociencias (CIN) y el Instituto de Investigaciones Psicológicas (IIP), ambos de la UCR, reportaron una antigüedad de 14 años y 54 años de existencia, respectivamente (ver tabla 3). Ambos albergaron entre 11-25 personas en Psicología que participaron en los procesos de investigación y reportaron la misma cantidad de personas colaborando que no fueron psicólogos. El CIN indicó que cuatro de sus docentes llevaron el título de licenciados en psicología y cuatro un doctorado. El IIP reportó cuatro psicólogos con bachillerato, 11 con licenciatura, cinco con maestría académica y 12 con doctorado. El personal no psicológico que participó en el CIN fueron: biólogos, biotecnólogos, genetistas, biólogos moleculares, estadísticos, ingenieros eléctricos, informáticos y diseñadores gráficos. Mientras en el IIP se encontraron estadísticos, biólogos, matemáticos, filólogos, lingüistas, médicos, fisiólogos y genetistas.

Todo el personal investigador de ambos centros colaboraron, también, en unidades académicas y se encontraron insertos en un régimen académico con más de 10 profesionales (CIN) y entre 11 y 25 (IIP) investigadores en Psicología con 6-10 y 21-25 proyectos de investigación activos, respectivamente. El enfoque metodológico reportado por el CIN fue cuasi-experimental y experimental y el IIP añadió instrumental y cualitativo con temáticas relacionadas con la Psicología.

En el CIN se encontraron investigaciones en la dimensión ambiental y desórdenes del estado de ánimo, ambiente colaborativo y funciones ejecutivas (en preescolares), memoria de trabajo y olvido, aprendizaje y funciones ejecutivas (EEG), cuidado maternal en relación con el aprendizaje y la memoria, ansiedad y estrés en relación con el aprendizaje y la memoria y condicionamiento al miedo (enriquecimiento ambiental y edad). Mientras el IIP reportó cognición social, psicometría y medición, desarrollo humano, procesos psicobiológicos, genética del comportamiento y comunicación de la ciencia.

El CIN indicó recibir financiamiento entre 300,000 a 5 millones de Colones (522 a 10,000 USD) para todos sus proyectos activos de investigación, provenientes de la Vicerrectoría de Investigación, Vicerrectoría de Acción Social y Centro de Investigación en Neurociencias (todos de la UCR), del Fondo de Estímulo a la Investigación, como premio ante diferentes entidades (como la Agencia Española de Cooperación Económica) y de los fondos para trabajos de graduación. El IIP reportó recibir para todos sus proyectos activos fondos de la Vicerrectoría de Investigación y de la Vicerrectoría de Acción Social (UCR) con 16 millones de Colones de fondos regulares (30,000 USD) y 10 millones de Colones de fondos concursables (19,000 USD), del Consejo Nacional de Rectores de Costa Rica (aprox. 30 millones de Colones (55,500 USD), el Consejo Nacional de Ciencia y Tecnología (CONICIT) y de los Fondos Fogarty (National Institute of Health, USA, aprox. 100,000 USD).

Los psicólogos del Centro de Investigación en Neurociencias se desarrollan en proyectos de neurobiología (trabajando principalmente con modelos animales) y de desarrollo cognitivo (trabajando principalmente con niños de preescolar) Sin embargo en los últimos años han ido incursionando también en los estudios genéticos y neuroquímicos en ambas áreas. Se desempeñan en un ambiente interdisciplinario de gran exigencia.

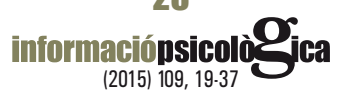


Tabla 3.- Datos de los programas de investigación en Psicología en Costa Rica, 2014, que respondieron la solicitud

\begin{tabular}{|c|c|c|}
\hline Ítem & $\begin{array}{l}\text { Centro de Investigación } \\
\text { en Neurociencias (CIN) }\end{array}$ & $\begin{array}{l}\text { Instituto de Investigacio- } \\
\text { nes Psicológicas (IIP) }\end{array}$ \\
\hline $\begin{array}{l}\text { 4. Cantidad de personal de investigación de } \\
\text { otras disciplinas con quienes colaboran los/as } \\
\text { investigadores/as de psicología. }\end{array}$ & Entre 11-25 & Entre 11-25 \\
\hline $\begin{array}{l}\text { 6. El personal investigador, también colabora } \\
\text { en unidades académicas. }\end{array}$ & Sí & Sí \\
\hline $\begin{array}{l}\text { 7. El personal investigador, produce investi- } \\
\text { gaciones (no necesariamente relacionadas con } \\
\text { el programa, instituto o centro de investiga- } \\
\text { ción), en las unidades académicas con las que } \\
\text { colabora. }\end{array}$ & No contesta & Sí \\
\hline $\begin{array}{l}\text { 8. Existe un régimen académico en el que está } \\
\text { inserto el personal investigador. }\end{array}$ & Sí & Sí \\
\hline $\begin{array}{l}\text { 13. Cantidad de artículos producidos por los/ } \\
\text { as investigadores/as psicólogos/as o con for- } \\
\text { mación psicológica. }\end{array}$ & Entre 2-10 & Entre 21-30 \\
\hline $\begin{array}{l}\text { 14. Cantidad de artículos aceptados en revistas } \\
\text { científicas indexadas. }\end{array}$ & Entre 2-10 & Entre 21-30 \\
\hline $\begin{array}{l}\text { 15. Las investigaciones del personal pasan } \\
\text { por un filtraje de consejo académico institu- } \\
\text { cional sobre metodología y ética investigativa } \\
\text { (Comité Ético Científicos Institucionales o } \\
\text { equivalentes). }\end{array}$ & Sí & Sí \\
\hline $\begin{array}{l}\text { 16. Cantidad de congresos/simposios/con- } \\
\text { ferencias en los que se han presentado los } \\
\text { resultados de las investigaciones consolidadas } \\
\text { o en curso. }\end{array}$ & Entre 11-15 & Más de 21 \\
\hline $\begin{array}{l}\text { 17. Existen sistemas o métodos de registro de } \\
\text { la producción científica del grupo (indicadores } \\
\text { de producción y calidad investigativa). }\end{array}$ & Sí & Sí \\
\hline \multirow[t]{4}{*}{$\begin{array}{l}\text { 19. Del total de proyectos activos, cuántos han } \\
\text { sido financiado por lo indicado en la Respues- } \\
\text { ta } 17 \text {. }\end{array}$} & \multirow[t]{4}{*}{ Entre 6-10 } & $\begin{array}{l}\text { Entre } 21-25 \\
\text { ( } 2 \text { de fondos especiales de } \\
\text { las vicerrectorías }\end{array}$ \\
\hline & & 2 de CONARE \\
\hline & & 1 CONICIT \\
\hline & & 1 Fogarty) \\
\hline $\begin{array}{l}\text { 21. Existen sistemas de entrenamiento y actua- } \\
\text { lización profesional del equipo investigador. }\end{array}$ & Sí & Sí \\
\hline
\end{tabular}




\section{Datos gestión}

La tabla 4 muestra los datos que maneja el Colegio Profesional de Psicólogos de Costa Rica (CPPCR) en relación a las publicaciones e investigación que se llevaron a cabo en Costa Rica. Reportó que existe un registro de la producción científica nacional en cuanto a artículos en revistas indexadas y presentaciones en congresos, entre otros. Según esta entidad, dicha información es un referente conceptual en la formación de profesionales en psicología.

Según el CPPCR, existe una regulación nacional (específica o general) de la investigación científica en Psicología desde tres aspectos: (a) La regulación desde el Código de Ética y Deontológico del mismo Colegio, (b) desde la legislación general sobre la investigación (Ley Reguladora de Investigación Biomédica, No. 9234) y animales (Ley de Bienestar de los Animales, No. 7451; Guía para el cuido y Uso de Animales de Laboratorio, Decreto MICIT No. 266668) y (c) la normativa de la Universidad de Costa Rica en cuanto a investigaciones con seres humanos (Reglamento Ético Científico de la Universidad de Costa Rica para las Investigaciones en las que Participan Seres Humanos y Reglamento de Cuido y Uso de Animales de Laboratorio).

En cuanto a la importancia de estas regulaciones en la investigación psicológica, el CPPCR informó que les permite regular y fiscalizar el ejercicio profesional y velar por la calidad de la investigación.

Según el registro del CPPCR (no necesariamente coincide con el CONESUP), 16 universidades en Costa Rica impartieron la carrera de Psicología, nueve que imparten posgrados en dicha carrera y tres que producen investigación científica. Alegó que no cuenta con un registro de la cantidad de agremiados que ejercen la docencia con grado de licenciatura, maestría o doctorado. Además, reportó que no existe un registro de la cantidad de agremiados en Psicología con nivel de maestría o licenciatura que desarrollen investigación científica. Y que a nivel nacional existe un posgrado de tipo académico productor de investigación científica pura y un posgrado de tipo profesional productor de investigación científica aplicada. Además, el Colegio Profesional de Psicólogos de Costa Rica asintió no contar con un registro de los montos de inversión nacional para la investigación científica.

Según informó el CPPCR, Costa Rica cuenta con dos programas de formación en Psicología acreditados por un sistema oficial, no así los posgrados. Esta institución no participa de estos sistemas de acreditación de las carreras, pues alegó que esto compete exclusivamente a las universidades y no al CPPCR. Por último, reconoció que no existe una Academia Nacional de Psicología que esté patrocinada por el ente gremial nacional.

El CPPCR no cuenta con registros sobre cantidad de profesionales agremiados con nivel de licenciatura, maestría o doctorado que ejercen la docencia universitaria o realizan investigación académica a nivel nacional. 
Tabla 4.- Datos sobre la Psicología nacional del Colegio Profesional de Psicólogos de Costa Rica

\section{Ítem}

1. Existe algún registro de la producción científica nacional en psicología (p.ej., artículos en revistas indexadas, premios en ciencia psicológica, presentaciones en congresos nacionales, regionales e internacionales).

2. ¿Cómo se utiliza la información de ese registro en la regulación y formación psicológica nacional?

3. Cantidad de revistas científicas de psicología incluidas en índices internacionales, bases de datos, repositorios u otros medios de recuperación de la producción científica.

6. Importancia de esa regulación en la investigación psicológica.

7. Cantidad de universidades que imparten la carrera (grado) de psicología en Costa Rica

8. Cantidad de universidades que imparten posgrados en psicología en Costa Rica.

9. Cantidad de universidades que producen investigación científica (artículos aceptados en revistas científicas indexadas internacionalmente) en psicología.

13. Cantidad de posgrados a nivel nacional con un enfoque de tipo académico y productor de investigación científica pura.

14. Cantidad de posgrados a nivel nacional con un enfoque de tipo profesional y productor de investigación científica aplicada.

15. Montos conocidos de inversión nacional en investigación científica psicológica (promedio para el periodo abarcado).

16. Cantidad de sistemas académicos de formación psicología (carreras, escuelas, facultades y posgrados) que están certificados en instituciones o grupos de acreditación nacional e internacional.
Colegio Profesional de Psicólogos de Costa Rica (CPPCR)
Al contar nuestro Colegio con una Revista Científica, se invita a la presentación de Ensayos y artículos científicos para efectos de su divulgación.

Esta información, permite acompañar la formación del profesional en Psicología como referente conceptual.

No contesta

Permite al Colegio como entidad reguladora y fiscalizadora del ejercicio profesional velar por el desarrollo de la calidad de la investigación en Psicología. Así como también proporcionar el producto al sector gremial con el fin de cultivar el desarrollo de líneas de trabajo político desde la Psicología.

No se cuenta con ese registro

Programas acreditados: 2

Programa de posgrados acreditados: 0 
17. El CPPCR participa en la acreditación de las unidades académicas.

patrocinada por el ente gremial nacional.
No

Al ser esta entidad meramente fiscalizadora y reguladora del ejercicio profesional, se estima la inconveniencia de ofrecer programas académicos, que quienes los aportan son las Universidades públicas y privadas.

\section{Datos de los currículos académicos}

En relación con los cursos de investigación impartidos durante la formación académica, los currículos de las ocho universidades revisadas a nivel de bachillerato, informaron que el $100 \%$ de las instituciones brindaron cursos de investigación con enfoques de tipo inductivo, hipotético deductivo y hermenéutico. A nivel de bachillerato, cuatro de las ocho universidades dictaron entre tres a siete cursos con contenidos orientados a la introducción en investigación, procesos cuantitativos y procesos cualitativos de investigación. A nivel de licenciatura, la mayor parte de las universidades revisadas ofrecieron un o dos cursos de investigación con contenidos mayoritariamente orientados a la preparación de los trabajos finales de graduación.

\section{Discusión}

De las 21 Escuelas de Psicología que existen en Costa Rica, solo se pudo recabar información de dos (UNIBE y UCR) a nivel de grado. En el nivel de posgrado, solo se pudo obtener información de la UCR. En conjunto, se pudo observar que existe un desarrollo en grados diferentes en cada una de estas universidades. La UCR cuenta con más de siete décadas de desarrollo universitario y, en lo que respecta a la Psicología, su Escuela tiene poco más de cuatro décadas de creación, fue la primera academia de formación de psicólogos/as en el país y desde ella se nutrieron las otras Escuelas de Psicología de universidades privadas y estatales. La UNIBE (privada) tiene una historia con alrededor de 16 años de existencia y una Facultad de Psicología de la misma edad.

Ahora bien, si se habla de los componentes académicos-investigativos de cada una de estas universidades, se debe considerar que la UCR no solo es la institución de educación superior más antigua de Costa Rica, sino que es la que cuenta con más recursos económicos, de personal, tecnología, facilidad de obtención de recursos para formación e investigación, contactos internacionales y nacionales, entre otras ventajas. Esto permite entender, por qué la diferencia en la cantidad de docentes que realicen investigación; a esto debe sumarse que varios de los docentes de la Escuela de Psicología de la UCR colaboran con otras unidades investigativas de la misma institución, lo que aumenta la diversidad académica y las conexiones de ese nivel.

En ese sentido, el Posgrado de Psicología de la UCR (UCR-SEP), que está adscrito al Sistema de Estudios de Posgrados de Costa Rica, se nutre de algunos docentes del grado y de

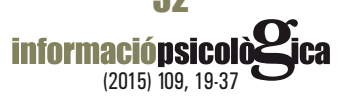


profesionales que proceden de otros contextos laborales. El posgrado posee un rango mayor de financiamiento para la investigación que la Escuela de Psicología.

La UNIBE (privada), si bien es una universidad joven, mantiene una Facultad de Psicología que alberga, aparte del grado en la disciplina, dos posgrados. Los procesos de investigación son recientes y comienzan a generar frutos con publicaciones y presencia en congresos. El financiamiento institucional para la investigación es menor, pues los recursos se captan directamente de los pagos de los estudiantes y no responden a los fondos públicos que recibe la UCR. A pesar de esto, es posible concursar en fondos internacionales para el uso en investigaciones locales, lo cual no siempre se logra resolver de manera exitosa para la universidad.

En general, se puede decir que la formación privada en Psicología en Costa Rica adolece de mecanismos de investigación consolidados; es difícil tener profesores/as a tiempo completo o parcial dentro del sistema académico dedicados a la investigación, asunto que, sí, es tradicional en las universidades estatales.

De manera estándar, la aparición del Sistema Nacional de Acreditación de la Educación Superior (SINAES) vino a generar una presión positiva sobre toda la educación superior costarricense. A partir del año 2002, se creó el SINAES por Ley (N. $\left.{ }^{\circ} 8256\right)$ que tiene por objetivo dar fe pública de la calidad académica de las instituciones, carreras y programas de educación superior (SINAES, 2014). Las universidades se pueden adherir de manera voluntaria e ingresar a un proceso de autoevaluación y evaluación por pares externos (por lo general, un profesional afín de Costa Rica y dos profesionales del extranjero). En el caso de la Psicología, la primera carrera que pasó todos los filtros de acreditación fue la UCR, luego siguió la Universidad Latina (quién no continuó los procesos posteriores de reacreditación), la Universidad Católica de Costa Rica continuó y aprobó el proceso, al igual que, más recientemente, la UNIBE. Al día de hoy, se mantienen acreditadas la UCR, la Universidad Católica de Costa Rica (que no envió información para este estudio) y la UNIBE. Uno de los componentes evaluados por el SINAES es la investigación y sus recomendaciones tienen un peso vinculante en la gestión presente y futura de las Escuelas, pues las obliga a mejorar sus actividades investigativas y aumentar la inversión en ese rubro (personal, material didáctico, tiempo de trabajo, recursos físicos, entre otros).

La investigación psicológica, al menos dentro del contexto de la formación de grado y posgrado, como lo indica la tabla 1, tiende a ordenarse, primero sobre los trabajos finales de graduación, luego sobre la colaboración de algunos de sus docentes en otros ámbitos más específicos del trabajo académico (institutos, centros de investigación) y, en menor medida, en programas o líneas de investigación autóctonos a las carreras de Psicología.

Algunos de los desarrollos han culminado en artículos científicos que se logran publicar en revistas indexadas, en otros casos (datos no presentados en este trabajo), solo logran ver la luz en revistas no indexadas. Dentro de esta línea de exposición, es necesario indicar que la Escuela de Psicología de la UCR colaboró en la creación de una revista psicológica (Wimb $l u$ ), organizada por los estudiantes del grado, que se encuentra en este momento indexada y es una de las tres revistas psicológicas de carácter científico que existen en Costa Rica.

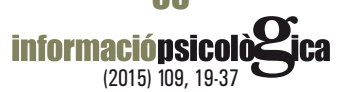


Por otra parte, la academia psicológica costarricense mantiene personal de la disciplina en varios tipos de programas, institutos o centros de investigación; sin embargo, solo se obtuvieron respuestas de dos lugares: el Instituto de Investigaciones Psicológicas (IIP) y el Centro de Investigación en Neurociencia (CIN), ambos de la UCR. Si bien existe otro centro de investigación en una universidad privada que tuvo éxito investigativo en el pasado, su casa de enseñanza no devolvió el cuestionario entregado.

De la misma forma en que se desarrolló la historia de la psicología en Costa Rica (Cordero, Dormond \& Flores, 2000; Thomas, 1979), ocurrió algo similar con la investigación científica en el país: La mayor parte de los/as profesionales en psicología con rango de doctorado académico se encuentran laborando a tiempo parcial o completo con la UCR. Esta institución ha sufragado, a su vez, parte o la totalidad de ese entrenamiento, que supone una reinserción en la misma institución con el consiguiente uso del conocimiento y habilidades dentro del sistema académico.

De manera específica, el IIP surge en la UCR como centro de investigación a las alturas de los años setenta y con el tiempo deviene en un instituto. Este instituto ha pasado por varias fases de desarrollo y es hoy un ente único en su tipo en la Psicología costarricense y contiene personal de psicología y de otros ámbitos con alta formación académica. Realiza investigaciones y obtiene fondos de alto calibre; su trabajo se ha orientado hacia una variada temática, por ejemplo: cognición social, psicometría y medición, desarrollo humano, psicobiología, genética del comportamiento y comunicación de la ciencia. Recibe financiamiento no solo de la UCR, sino de entes como el National Institute of Health. Los/as investigadores/as fungen, algunos de ellos, como docentes en la Escuela de Psicología de la UCR y existe una participación en simposios, congresos y actividades académicas internas a la UCR e internacionales. Es importante mencionar, que el IIP administra una revista científica indexada (Actualidades en Psicología), que ha logrado posicionarse como referente en el ámbito de la Psicología. La misma pasó de ser un documento monográfico (durante los años ochenta y noventa) a una revista con las características esperadas a nivel internacional.

En cambio, el CIN expresa una visión mucho más focalizada en la Neurociencia; sin embargo, en los últimos años ha incursionado en estudios genéticos y neuroquímicos y entre su personal se cuenta con psicólogos/as, muchos/as de estos/as cuentan con una preparación académica de tipo doctoral. El CIN mantiene lazos colaborativos con instituciones extranjeras y nacionales, de forma específica tiene vínculos directos con el IIP. Su personal es tan variado como el del IIP, lo cual dota su trabajo de una gran riqueza académica.

Hasta hace unos cinco años, el CIN tenía un rango de programas, pero ha contado con apoyo de la Vicerrectoría de Investigación de la UCR y otros cargos académicos de alta envergadura y de forma fundamental. Gracias a su trabajo sistemático y organizado, pudo escalar a un Centro de investigación, que recibe fondos propios de la UCR, pero también concursa por recursos internacionales.

Ambos entes, el IIP y el CIN, se han convertido en hitos para la investigación científica psicológica costarricense. Esta investigación tiene claro la existencia de otros grupos en la misma UCR, en la Universidad Nacional y la Universidad Estatal a Distancia (estas dos 
últimas, también, universidades públicas, tampoco proveyeron información sobre su accionar) con tendencias en sus enfoques investigativos. En cuanto a los enfoques investigativos, llama la atención que no se representan (sobre todo en el caso del IIP) ámbitos como la Psicología clínica, la Psicología de la salud, Psicología del deporte, la Psicología de las organizaciones o la Psicología forense, por mencionar temas de valor para Costa Rica. Por lo general, las acciones investigativas han respondido más bien a un área de interés particular o a proyectos establecidos de acuerdo con líneas de trabajo de interés para el ente como tal. No es claro, si, en general, esas áreas de inserción actuales y las no presentes, responden o podrían responder a áreas de trabajo prioritario para la vida nacional; pero, sí, es un hecho que se alinean con los estándares y líneas de investigación internacionales.

El Colegio Profesional de Psicólogos de Costa Rica (CPPCR) facilitó información sobre su quehacer y la regulación disciplinar nacional. El CPPCR está facultado por ley para convertirse en la rectoría de la actividad profesional, con el fin de proteger a la ciudadanía de la actividad profesional de la psicología. Esto implica acciones afirmativas para regular la disciplina. Sin embargo, su enfoque no ha sido uno de tipo académico, sino fiscalizador. Con el tiempo, se han creado lineamientos y estructuras que han procurado brindar apoyo en capacitación, formación y divulgación. Es importante mencionar esto, pues el CPPCR reúne a casi todos/as los/as profesionales de la Psicología costarricense, muchos de las y los cuales colaboran en las escuelas, facultades, posgrados, institutos y centros que se han mencionado en esta investigación y en otros no incluidos.

Igualmente, el CPPCR creó, hace poco más de 30 años una revista (Revista Costarricense de Psicología), que con el paso del tiempo se ha transformado en una de carácter científico, al organizar su accionar para facilitar (dentro de los estándares internacionales) la comunicación científica a los/as psicólogos/as nacionales y extranjeros.

Sin embargo, el CPPCR no tiene un registro de investigación psicológica, si bien conoce la cantidad de Escuelas de Psicología en funcionamiento, no puede intervenir directamente en ellas, pues están reguladas por otros organismos de educación superior independientes. Esto incurre en que la formación psicológica costarricense no mantenga líneas comunes en cuanto a las necesidades curriculares y competenciales que requiere el país, que, a su vez, incide en que no es posible hablar de líneas maestras de temas de investigación, pues tampoco se conocen.

A modo de corolario, puede indicarse que Costa Rica ha tenido un desarrollo de la psicología, que ha pasado de una de corte academicista y asistencialista (los llamados aplicadores de pruebas), con estados intermedios de búsqueda de identidad, a un momento actual, que procura desarrollar enfoques más sistemáticos y consolidados de formación y entrenamiento. Aquí, la creación del SINAES se ha vuelto un punto de inflexión, desde el cual se han manifestado cambios en el enfoque de entrenamiento y de comprensión de lo psicológico, sobre los debates de qué es ciencia y la importancia de generar investigación de calidad científica.

Costa Rica se enfrenta a una serie de necesidades en salud, educación, criminalidad, desarrollo organizacional, política, gobernanza, diversidad y otros temas vitales, ante los cuales 
la psicología debe posicionarse. Visto así, el futuro es promisorio y retador, una promesa de que la psicología costarricense se puede insertar en áreas antes no exploradas, si se realizan los ajustes necesarios; y es retador, en la medida que solo al romper esquemas antiguos y algo desactualizados de formar, se podrá afrontar el porvenir.

De las 21 Escuelas de Psicología, solo se pudo dar cuenta de dos que realizan un trabajo en esa dirección; de 21 universidades, solo una posee programas de investigación formales y altamente desarrollados. La historia demuestra que, cuando se deja de lado la investigación científica en los programas de formación, se pierde el sentido de la academia y se pasa a convertir en un simple creador de profesionales, pero no de científicos/as y pensadores/as, que puedan cambiar el porvenir de una nación.

Para terminar, una pregunta que trasciende lo investigado: ¿Es lícito hacer, inclusive, la pregunta del control de la calidad y el avance de la Psicología en Costa Rica en el contexto latinoamericano a la luz de los procesos de globalización y migración? La Unión Europea (UE) creó en el año 1999 en la "Declaración de Bologna" un sistema de educación superior integrado (Bachelor-Master-System) con estructuras que garanticen la calidad interna y externa y que integra actualmente a 47 naciones. Los tres objetivos principales de esta declaración encerraron (a) la movilidad de la educación universitaria dentro de la UE, (b) la competitividad internacional y (c) la empleabilidad. Estos objetivos se concretaron por medio de la promoción de dimensiones dirigidas a: (a) La fácil comprensión y comparación de los grados académicos, (b) el aseguramiento de la calidad con criterios y metodologías comparables, (c) la transmisión de competencias interculturales para aumentar la movilidad internacional por medio de un desarrollo curricular y de programas de estudio en cooperación entre instituciones, (d) la integración de la formación y la investigación y (e) el aumento de la atractividad del espacio académico (universitario) europeo, al establecer el principio de "aprendizaje de por vida". Este proceso implicó, p.ej., el desarrollo de nuevas definiciones de las carreras y de nuevos reglamentos y programas de estudios que se adecuaren al estándar europeo sin poner en peligro la academia local.

Además, el Proceso Bologna se encuentra apoyado por el Bologna-Follow-Up-Group, que se constituye a nivel europeo por integrantes de los ministerios de cultura, educación e investigación; intercambios académicos, conferencias de rectores de educación superior, asociaciones de estudiantes, asociaciones de empresarios, etc., quienes reciben a nivel de su país regularmente seminarios con ayuda concreta para implementar el proceso en su institución. Adicionalmente, se enfatizaron en el proceso la dimensión de la justicia social y cultural en el estudio, lo que incluye la evaluación de su financiamiento, el tiempo y recursos para pasantías, la necesidad de aumentar becas y la mejora de las oportunidades laborales, de las bases de datos y de la información. En el ámbito de los estudios y de la enseñanza surgieron diferenciaciones entre funciones antiguas, como la planeación del desarrollo, dirección de los intercambios estudiantiles, orientación en los estudios, elección y funciones de los decanos de las facultades, etc., y funciones nuevas, como la acreditación, la evaluación de la enseñanza, el diseño curricular (basado en la evidencia), el E-Learning, la movilidad estudiantil, etc. 
En el esfuerzo de evaluar la academia costarricense, resulta hoy tanto como en su propia historia imposible dejar por fuera criterios de internacionalidad y de control de calidad interna y externa, que se encuentran íntimamente asociados a la capacitación continua del personal docente y administrativo. ¿Un ejemplo modelo cuya traducción queda pendiente para la región latinoamericana?

Antes dar cierre a la discusión se debe indicar que hubo una serie de limitaciones en el estudio, entre ellas el bajo número de participantes institucionales a nivel de escuelas de psicología lo que, para este caso, afectó las posibilidades de extrapolar la información a todas las universidades públicas y privadas de Costa Rica. En el caso de los centros e institutos de investigación en psicología, solo se pudo obtener respuesta de los dos directamente vinculados a esta disciplina.

\section{Referencias}

Adis, G. (Diciembre, 1991). Sobre la historia de la Psicología Clínica en Costa Rica. Revista Cúpula, 27, $30-34$.

Adis, G. (Junio, 1994). La Psicología en la Universidad de Costa Rica: Apuntes históricos. Revista de Ciencias Sociales, 64, 17-26

Calvo Cerdas, C., Charpentier Álvarez, D., Fallas Arias, J., Jiménez Acuña, J., Rivera Dittel, A. C. y Torres Solano, J. (2010). Historia de la Psicología costarricense, Período 1957-1985 (Memoria de Seminario de graduación para optar por el grado de Licenciatura en Psicología). Universidad de Costa Rica, Facultad de Ciencias Sociales, Escuela de Psicología, Ciudad Universitaria Rodrigo Facio, San José, Costa Rica.

Cordero Cordero, T. y Salas Calvo, J. M. (2003). La Psicología en Costa Rica. Encrucijada en el nuevo milenio. Revista Interamericana de Psicología, 37(1), 93-108. Recuperado de http://www.redalyc.org/articulo. oa?id=28437106

Cordero Fernández, O. M., Dormond Sánchez, S. y Flores Mora, D. (2000). Historia y perspectivas de la Psicología en Costa Rica. En Desarrollo de la Psicología en Latinoamérica. (pp. 25-58). Recuperado de http://www. coedu.usf.edu/zalaquett/formacion II/costarica.pdf

Consejo Nacional de Enseñanza Superior Universitaria Privada (2013). Memoria de labores del período 2007-2012. San José, Costa Rica: Autor.

Consejo Nacional de Rectores de Costa Rica (2010). Seguimiento de la condición laboral de las personas graduadas 2000-2007 de las Universidades Costarricenses. San José, Costa Rica: Autor/ Oficina de Planificación de la Educación Superior/ Observatorio Laboral de Profesiones. Recuperado de http://ucrcomunicacion.wdfiles.com/local--files/start/Seguimiento\%20a\%20situacion\%20laboral

Díaz, D. y Díaz, C. (2014). Ponencia: El Sistema de Estudios de Posgrado en la estructura organizacional de la Universidad de Costa Rica. VIII Congreso Universidad de Costa Rica, San José, Costa Rica. Recuperado de http://setimocongreso.ucr.ac.cr/sites/default/files/ponencias/EGH-26.pdf

Programa Estado de la Nación (2013). Cuarto Informe sobre el Estado de la Educación. San José, Costa Rica: Autor. Recuperado de http://www.estadonacion.or.cr/estado-educacion/educacion-informe-ultimo

Sistema Nacional de Acreditación de la Educación Superior (2014). ¿Qué es el SINAES? San José, Costa Rica: Autor. Recuperado de http://www.sinaes.ac.cr/index.php/que-es-el-sinaes

Thomas, P. (1979). El desarrollo de la Psicología en Costa Rica. En Acuña Sanabria, J y colaboradores, La Psiquiatría y la Psicología en Costa Rica (pp. 43-76). San José, Costa Rica: Editorial Universidad Estatal a Distancia 